\title{
SIMPLIFYING THE FEEDING NETWORK FOR MULTI- BEAM CIRCULAR ANTENNA ARRAYS BY USING CORPS
}

\author{
M. A. Panduro \\ Unidad Académica Multidisciplinaria Reynosa-Rodhe \\ Universidad Autónoma de Tamaulipas (UAT) \\ Carretera Reynosa-San Fernando, Reynosa, Tamaulipas 88779, México \\ C. del Rio-Bocio \\ Universidad Pública de Navarra \\ Campus Arrosadia, Pamplona 31006, España
}

\begin{abstract}
An innovative way to analyze the design of beam-forming networks (BFN) for scannable multi-beam circular antenna arrays using the CORPS (Coherently Radiating Periodic Structures) concept is introduced. This design of CORPS-BFN considers the optimization of the complex inputs of the feeding network by using the Differential Evolution (DE) method. Simulation results for different configurations of CORPS-BFN for a scannable circular array are presented. The results shown in this paper illustrate certain interesting characteristics in the behavior of the array factor for the scannable circular array. The most significant aspect that is unique to this proposal is the simplification of the feeding network based on CORPS.
\end{abstract}

\section{INTRODUCTION}

The flexibility and re-configurability are two important features of the present and future antenna systems. These two properties could be very easily defined combining smartly different independent beams or signals of the same antenna system. These systems, which are capable of managing independently different beams, are usually referred to as multi-beam systems. These systems make use of different techniques that determine the complex excitations of the different radiating elements to be provided by the beam forming network.

Received 2 January 2011, Accepted 4 March 2011, Scheduled 6 March 2011

Corresponding author: Marco A. Panduro (mamendoza@uat.edu.mx). 
There are different alternatives to use a feed network. In this research work, it is presented the concept of the principles of CORPS [1-3] in a circular antenna array. This is in order to design a beam-forming network for a multi-beam antenna system.

In order to set new design philosophies, the CORPS concept has firstly been applied directly to the radiating elements [4]. The design of BFN for scannable multi-beam linear arrays using CORPS was presented in [1] and [3]. The result of these works revealed that the design of CORPS-BFN optimizing the complex inputs with evolutionary optimization could generate scannable multiple beams for a linear array with a significant simplification of the feeding network. However, the results presented in [1] and [3] consider only the simple case of a linear array. The application of the CORPS technology for scannable multi-beam bi-dimensional arrays and the study of new structures for designing BFN could generate design cases where the simplification of the feeding network is still more significant with respect to the reported results.

In our case, it will introduce an innovative way to analyze the design of BFN for scannable multi-beam circular antenna arrays using the CORPS concept. The main objective of this paper is to combine the technology based on CORPS to define the BFN and the DE [510] method to look for optimal excitations, in order to generate a scannable multi-beam circular array. The contribution of this paper is to present a perspective of the design of CORPS-BFN considering scannable multi-beam circular arrays.

\section{BEHAVIOR OF THE CORPS-BFN}

A schematic representation of a CORPS-BFN of $n$ inputs, $N$ outputs and 3 layers is presented in Fig. 1. As shown in Fig. 1, a CORPSBFN is set by a mesh interconnected by means of Split $(\mathrm{S})$-nodes and Recombination (R)-nodes. The CORPS-BFN works as follows: the signal entered by one input port is divided in two and added with the arriving signals of the neighboring input ports. Following the path of each signal, we will find something like an inverted triangle which has the lower vertex at the input port. The opposite side of this vertex will define the output ports receiving some information from this input port, or in other words, the effective radiating area from which every input signal (or orthogonal beam) will be radiated. Since the isolation between the input ports is ensured and the spreading of the signal inside the structure is controlled, the CORPS-BFN is able to handle simultaneously several orthogonal beams without any problem. In the outermost branches, the inputs that are not used are finished with a matched load in order to avoid reflections. 


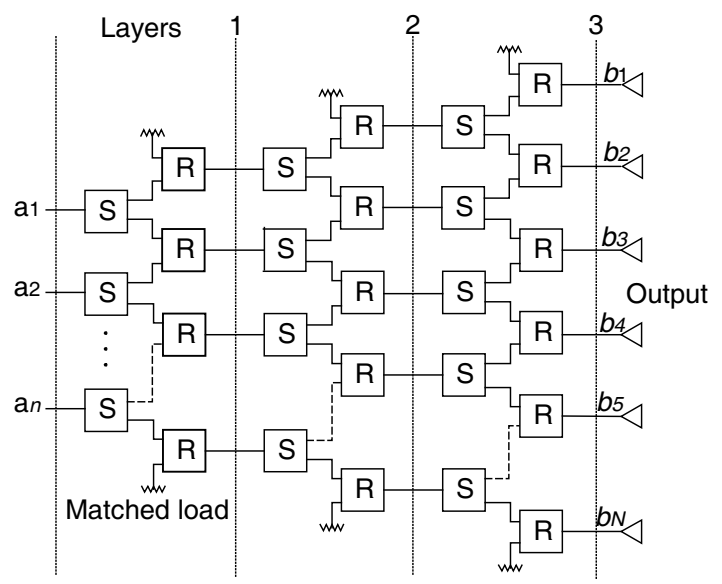

Figure 1. Schematic representation of a CORPS-BFN with $\mathrm{S}$ and $\mathrm{R}$ nodes. Reproduced courtesy of The Electromagnetics Academy

From [1] and [2], the Unitary Cell Scattering matrix that represents the behaviour of an S-node could be extracted as follows:

$$
[\mathrm{S}]=\left[\begin{array}{lll}
0 & j / \sqrt{2} & j / \sqrt{2} \\
j / \sqrt{2} & 0 & 0 \\
j / \sqrt{2} & 0 & 0
\end{array}\right]
$$

In the same way, in order to evaluate the fields after an S-node or R-node the next expression can be used

$$
V^{-}=S V^{+}
$$

In (2), $S$ is the Scattering Matrix of an S-node and $V^{+}$is the Amplitude and Phase of the field at input ports of an S-node. Using (2) and the schematic representation of a CORPS-BFN (Fig. 1) it is possible to establish an iterative code (i.e., with MATLAB) that represents the propagation of signal throughout a general configured CORPS-BFN.

It is possible to establish different configurations for the CORPSBFN with different number of inputs, outputs and layers. In this case, several orthogonal beams could be generated simultaneously by intercalating the inputs of the CORPS-BFN, i.e., a group of different inputs will generate the beam \#1 and another group of inputs could generate the beam \#2. Following the philosophy of CORPS, each group of inputs must be established in a strategic way in order to have the capability to control electronically the corresponding beam pattern with a smaller number of complex inputs with respect to the number of antenna elements employed. To set an example, the next configurations could be of interest. 
1) For a system of 26 radiators and 25 input ports (a CORPS-BFN of one layer) two orthogonal beams could be generated simultaneously by intercalating the inputs of the CORPS-BFN, as shown in the Fig. 2. It could be observed that the input of each antenna element is the output of an R-node (of the last layer), that combines the outputs of the two neighbouring S-nodes. So, it is possible to control two antenna elements with one complex input. The interesting aspect of this case is that the group of 13 inputs (that generates the beam \#1) could control the 26 radiators of the array, and the remaining 12 (used for the beam \#2) could control to 24 of them.

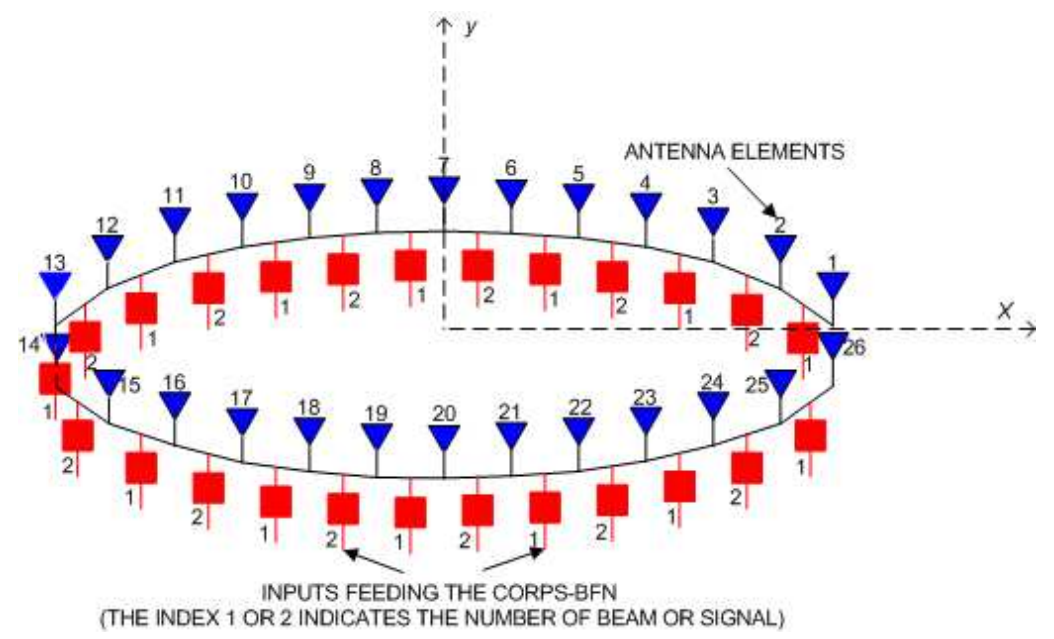

Figure 2. System of 26 radiators and 25 input ports. Reproduced courtesy of The Electromagnetics Academy

2) For the system of 26 radiators, we could use a CORPS-BFN of two layers with 24 input ports. Two orthogonal beams could be generated simultaneously by intercalating the inputs of the CORPSBFN by pairs as illustrated in the Fig. 3. In this case, it could be observed that a pair of complex inputs could control four antenna elements. In the case of the beam \#1, 12 of 24 input ports could control 24 radiators of the array. For the beam \#2, the remaining 12 input ports control to 24 of 26 radiators.

For a set of complex inputs $[\mathbf{a}]$ feeding the CORPS-BFN, as shown in Fig. 1, the characteristics of Directivity $(D)$ and Side Lobe Level $(S L L)$ for each beam pattern can be calculated using the equation of the array factor as $[7,11]$

$$
A F(\phi, \mathbf{a})=\sum_{n=1}^{N} b_{n} \exp \left[j k r\left(\cos \left(\phi-\Delta \phi_{n}\right)-\cos \left(\phi_{0}-\Delta \phi_{n}\right)\right)\right]
$$




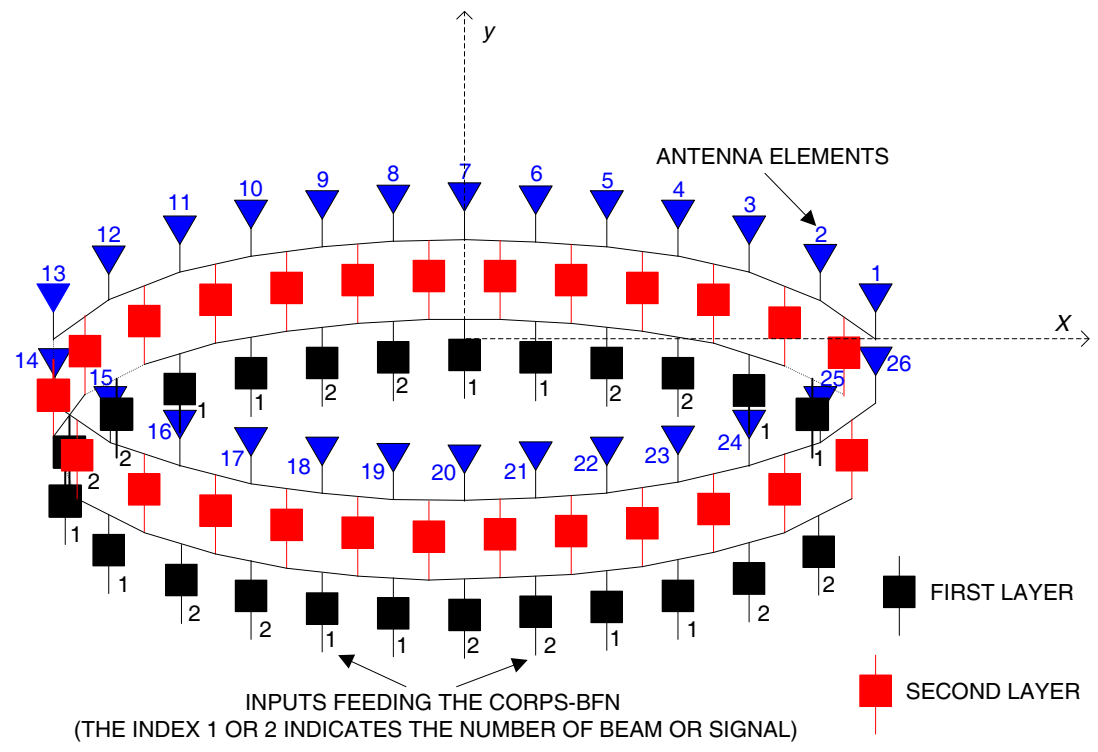

Figure 3. System of 26 radiators and 24 input ports. Reproduced courtesy of The Electromagnetics Academy

where $b_{i}$ represents the complex excitation of the $i$ th antenna element of the array, $\Delta \phi_{n}=2 \pi(n-1) / N$ for $n=1,2, \ldots, N$ is the angular position of the $n$th element on the $x-y$ plane, $k r=N d$, i.e., $r=$ $N d \lambda / 2 \pi, \phi_{0}$ is the direction of maximum radiation and $\phi$ is the angle of incidence of the plane wave.

Next, the objective function and the technique used are described.

\section{OBJECTIVE FUNCTION AND THE TECHNIQUE USED}

The most prominent advantage of $\mathrm{DE}$ is its low computation time compared to Genetic Algorithms (GA) [12-14]. DE is an alternative to speed up the GA.

For each vector or solution (amplitude and phase of the complex inputs feeding the CORPS-BFN) of the population $\left(N_{p}\right) X_{i}, i=$ $1,2, \ldots, N_{p}$ of the $G_{t h}$ iteration, two new trial members, $\varepsilon_{t 1}$ and $\varepsilon_{t 2}$, are generated as follows:

$$
\begin{aligned}
& \varepsilon_{t 1}=\varepsilon_{r 1}^{(G)}+F\left(X_{i}^{(G)}-\varepsilon_{r 2}^{(G)}\right) \\
& \varepsilon_{t 2}=\varepsilon_{r 1}^{(G)}+F\left(X_{i}^{(G)}-\varepsilon_{r 3}^{(G)}\right)
\end{aligned}
$$


where $F \in[0,2]$ is a real constant factor range suggested in [8], which controls the amplification of the differential variation, and the integers $r_{1}, r_{2}, r_{3} \in\left[1, N_{p}\right]$ are randomly chosen such that $r_{1} \neq r_{2} \neq r_{3}$.

In this case, each individual generates an array factor of certain characteristics of $S L L$ and $D$. Therefore, the design problem is formulated as minimize the next objective function

$$
\text { Obj-fun }=\left(\left|A F\left(\phi_{S L L} \mathbf{a}\right)\right| / \max |A F(\phi, \mathbf{a})|\right)+(1 / D(\phi, \mathbf{a}))
$$

where $\phi_{S L L}$ is the angle where the maximum side lobe is attained. In this case, both objectives ( $S L L$ and $D$ ) are uniformly weighted in the cost function.

After the objective function evaluation, the best solution in the set $\left\{\varepsilon_{i}, \varepsilon_{t 1}, \varepsilon_{t 2}\right\}$ becomes the new member for the next iteration, $\varepsilon_{i}^{G+1}$. Some chromosomes in the new population occasionally generate array factors which are not physically realizable, and an adjusting process is needed [6]. A termination criterion is proposed by fixing a number of iterations without an improvement over this solution. In $[5,6]$, it is explained the procedure of DE in detail.

The simulation results of using this evolutionary algorithm are presented in the next section.

\section{SIMULATION RESULTS}

The DE algorithm was implemented to study the behavior of the array factor generated by the configurations shown in the Section 2 . The experiments parameters were set as follows: maximum number of generations $r_{\max }=500$, population size $N_{p}=200$, and $F=0.5$ [5].

Figures 4-5 illustrate the behavior of the array factor generated by the configurations shown in Fig. 2 and Fig. 3. As shown in the Figs. 45 , two simultaneous beams of different signals could be generated by such CORPS-BFN. These figures illustrate that the amplitude and phase excitations feeding the CORPS-BFN optimized by the DE algorithm can achieve a good performance in the two beams. In addition to, it is observed that this performance is remained as the main beam of different signals is scanned in the azimuth plane.

If it is considered the beam 1 from the Fig. 4 , it is obtained a $S L L=-12.66 \mathrm{~dB}$ and $D=13.62 \mathrm{~dB}$ for $\phi_{0}=30^{\circ}, S L L=-11.95 \mathrm{~dB}$ and $D=13.55 \mathrm{~dB}$ for $\phi_{0}=150^{\circ}$, and $S L L=-11.71 \mathrm{~dB}$ and $D=13.54 \mathrm{~dB}$ for $\phi_{0}=270^{\circ}$. For the beam 2, it is obtained a $S L L=$ $-12.21 \mathrm{~dB}$ and $D=13.57 \mathrm{~dB}$ for $\phi_{0}=90^{\circ}, S L L=-11.8 \mathrm{~dB}$ and $D=13.4 \mathrm{~dB}$ for $\phi_{0}=210^{\circ}$, and $S L L=-11.92 \mathrm{~dB}$ and $D=13.51 \mathrm{~dB}$ for $\phi_{0}=330^{\circ}$.

Considering the beam 1 from the Fig. 5, it is obtained a $S L L=$ $-12.22 \mathrm{~dB}$ and $D=13.44 \mathrm{~dB}$ for $\phi_{0}=30^{\circ}, S L L=-12.58 \mathrm{~dB}$ 


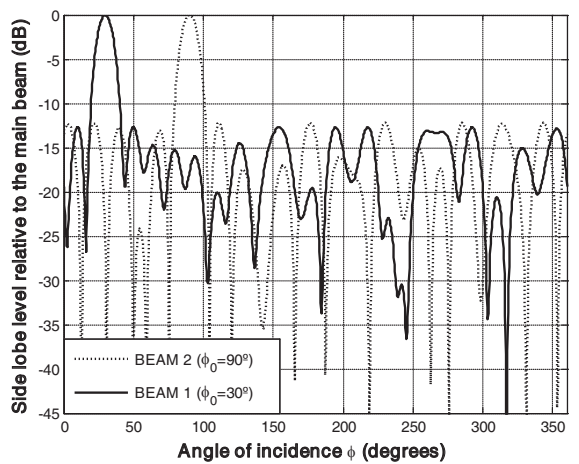

(a)

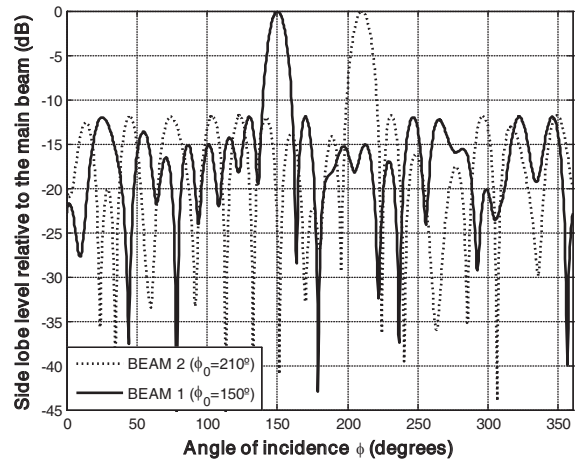

(b)

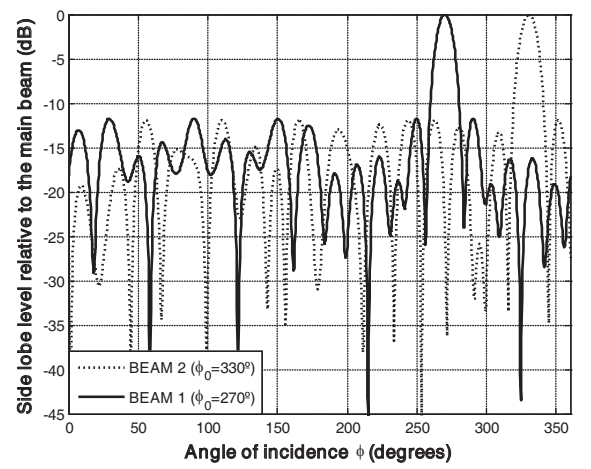

(c)

Figure 4. Array factor generated by the configuration 1 shown in Fig. 2, (a) $\phi_{0}=30^{\circ}$ for beam \#1 and $\phi_{0}=90^{\circ}$ for beam \#2, (b) $\phi_{0}=150^{\circ}$ for beam $\# 1$ and $\phi_{0}=210^{\circ}$ for beam $\# 2$, (c) $\phi_{0}=270^{\circ}$ for beam $\# 1$ and $\phi_{0}=330^{\circ}$ for beam $\# 2$.

Reproduced courtesy of The Electromagnetics Academy

$D=13.44 \mathrm{~dB}$ for $\phi_{0}=150^{\circ}$, and $S L L=-12.47 \mathrm{~dB}$ and $D=13.49 \mathrm{~dB}$ for $\phi_{0}=270^{\circ}$. For the beam 2, it is obtained a $S L L=-12.53 \mathrm{~dB}$ and $D=13.49 \mathrm{~dB}$ for $\phi_{0}=90^{\circ}, S L L=-12.23 \mathrm{~dB}, D=13.46 \mathrm{~dB}$ for $\phi_{0}=210^{\circ}$, and $S L L=-12.63 \mathrm{~dB}$ and $D=13.45 \mathrm{~dB}$ for $\phi_{0}=330^{\circ}$.

If these results are compared with respect to the uniform excitation case with conventional progressive phase excitation for $N=26(S L L=-6.92 \mathrm{~dB}$ and $D=13.21 \mathrm{~dB})$, we have a very significant performance improvement in terms of the side lobe level and a very significant simplification of the feeding network. In this case, the interesting aspect is that these two scannable beams are generated with $N / 2$ complex inputs, i.e., the relationship between the number of complex inputs $n$ and the number of antenna elements $N$ for these 


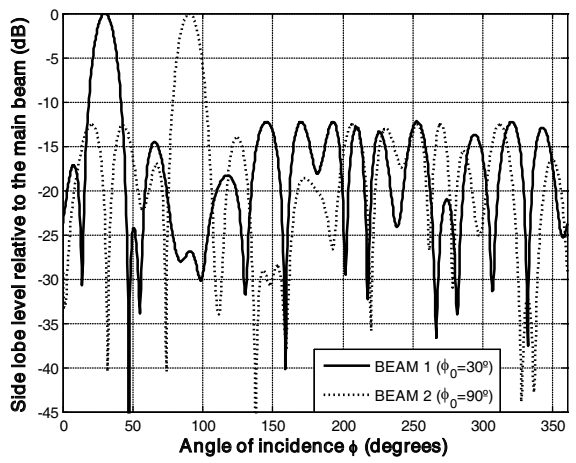

(a)

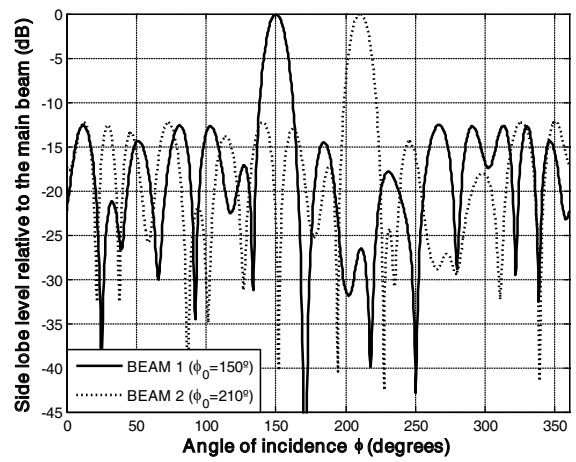

(b)

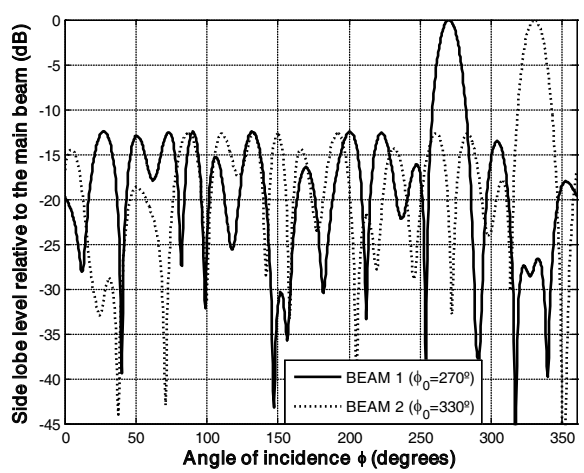

(c)

Figure 5. Array factor generated by the configuration 2 illustrated in the Fig. 3 , (a) $\phi_{0}=30^{\circ}$ for beam $\# 1$ and $\phi_{0}=90^{\circ}$ for beam $\# 2$, (b) $\phi_{0}=150^{\circ}$ for beam \#1 and $\phi_{0}=210^{\circ}$ for beam \#2, (c) $\phi_{0}=270^{\circ}$ for beam \#1 and $\phi_{0}=330^{\circ}$ for beam \#2.

\section{Reproduced courtesy of The Electromagnetics Academy}

particular configurations is $N / 2$, or $n \cdot 1$ CORPS layer $=N / 2$ for configuration 1 (illustrated in Fig. 2) and $n \cdot 2$ CORPS layer $=N$ for the second configuration illustrated in Fig. 3.

In this case, it is illustrated the scanning capability in angles of $\left\{30^{\circ}, 150^{\circ}, 270^{\circ}\right\}$ for beam 1 and $\left\{90^{\circ}, 210^{\circ}, 330^{\circ}\right\}$ for beam 2. However, the overall performance (directivity, $S L L$ ) would not be affected by the angle between the beams or other angles different to the cases presented in previous examples.

In this paper, the idea was to demonstrate the possibilities of simplifying the feeding network for multi-beam circular antenna arrays by using CORPS. Although it was presented the case to generate two scannable beams, it is perfectly possible to define independently the 
number of input ports (defined by the number of orthogonal beams to be used simultaneously) and the number of radiating elements. Furthermore, although only simulation results were presented in the paper, flexible substrates could be a practical consideration for a possible implementation of this circular topology.

\section{CONCLUSION}

The design of beam-forming networks for scannable multi-beam circular antenna arrays using CORPS has been presented. Simulation results reveal that the design of CORPS-BFN optimizing the complex inputs with the DE algorithm could generate scannable multiple beams with a significant simplification of the feeding network. The behavior of the array factor for different configurations of CORPS-BFN for a scannable multibeam circular array was studied and analyzed.

\section{ACKNOWLEDGMENT}

This work was supported by the Mexican National Science and Technology Council, CONACyT, under grant 127919 and the Science and Technology Council of Tamaulipas Mexico COTACyT under grant 2007-C13-73901 and 108166.

\section{REFERENCES}

1. Panduro, M. A. and C. del Rio-Bocio, "Design of beam-forming network using CORPS and evolutionary optimization," Int. J. Electron. Commun. (AEU), Vol. 63, 353-365, 2009.

2. Betancourt, D., A. Ibañez, and C. del Rio-Bocio, "A novel methodology to feed phased array antennas," IEEE Trans. Antennas Propagation, Vol. 55, 2489-2494, 2007.

3. Panduro, M. A. and C. del Rio-Bocio, "Design of beam-forming network for scannable multi-beam antenna arrays using CORPS," Progress In Electromagnetics Research, Vol. 84, 173-188, 2008.

4. Betancourt, D., A. Ibañez, and C. del Rio-Bocio, "Coherently radiating periodic structures (CORPS): A step towards highresolution radiations systems," IEEE AP-S 2005 Washington, DC, 2005.

5. Feortisov, V. and S. Janaqui, "Generalization of the strategies in differential evolution," Proceedings of the IEEE Conference Evolutionary Computation, 1996. 
6. Yang, S., A. Qing, and Y. B. Gan, "Synthesis of low side lobe antenna arrays using the differential evolution algorithm," IEEE Transactions on Antennas and Propagation Conference, 1-22, 2003.

7. Panduro, M. A., C. A. Brizuela, L. I. Balderas, and D. A. Acosta, "A comparison of genetic algorithms, particle swarm optimization and the differential evolution method for the design of scannable circular antenna arrays," Progress In Electromagnetics Research B, Vol. 13, 171-186, 2009.

8. Kurup, D., M. Himdi, and A. Rydberg, "Synthesis of uniform amplitude unequally spaced antenna arrays using the differential algorithm," IEEE Trans. Antennas Propagation, Vol. 51, 22102217, 2003.

9. Parsopoulos, K. E., D. K. Tasoulis, N. G. Pavlidis, V. P. Plagianakos, and M. N. Vrahatis, "Vector evaluated differential evolution for multi-objective optimization," IEEE Congress on Evolutionay Computation, 19-23, 2004.

10. Storn, R. and K. Price, "Minimizing the real functions of the ICEC'96 contest by differential evolution," IEEE Congress on Evolutionay Computation, 1996.

11. Balanis, C., Antenna Theory - Analysis and Design, 2nd Edition, Wiley, New York, 1997.

12. Rahmat-Samii, Y. and E. Michielsen, Electromagnetic Optimization by Genetic Algorithms, Wiley \& Sons, New York, 1999.

13. Haupt, R., "Thinned arrays using genetic algorithms," IEEE Transactions on Antennas and Propagation, Vol. 42, 993-999, 1994.

14. Goldberg, D. E., Genetic Algorithms in Search, Optimization and Machine Learning, Addison-Wesley, Massachusetts, 1989. 